\title{
Combinatorial Generation of Small Point Configurations and Hyperplane Arrangements
}

\author{
Lukas Finschi \\ Komei Fukuda
}

\begin{abstract}
A recent progress on the complete enumeration of oriented matroids enables us to generate all combinatorial types of small point configurations and hyperplane arrangements in general dimension, including degenerate ones. This extends a number of former works which concentrated on the non-degenerate case and are usually limited to dimension 2 or 3 . Our initial study on the complete list for small cases has shown its potential in resolving geometric conjectures.
\end{abstract}

\section{Introduction}

The generation of combinatorial types of point configurations and hyperplane arrangements has long been an outstanding problem of combinatorial geometry. A point configuration is a set of $n$ points in the real Euclidean space $\mathbb{R}^{d}$. Its combinatorial type, called order type, is determined by the relative positions of the points, more formally by the set of all partitions of the $n$ points by hyperplanes, where the points may be arbitrarily relabeled. Similarly, a hyperplane arrangement is a set of $n$ affine hyperplanes in $\mathbb{R}^{d}$, and its combinatorial type, which we call its dissection type, is determined by the relative positions of all cells. For the generation of these combinatorial types no direct method is known, and it appears to be necessary to use combinatorial abstractions - allowable sequences of permutations, $\lambda$-functions, chirotopes, combinatorial geometries, or oriented matroids; in our work we will use oriented matroids [BLVS $\left.{ }^{+} 99\right]$. These abstractions are more general than their geometric counterparts, e.g., there exist oriented matroids which cannot be realized by any point configuration. Although it is NP-hard to decide whether a given oriented matroid is realizable or not [Mnë88, Sho91] the realizability problem is decidable and there are practical methods which work satisfactory for small instances, at least in the non-degenerate case (e.g., see [RG92]).

The former work on generation of point configurations and related struc- 
tures (e.g., see [GP83, GP84, BGdO00, AAK01]) concentrated on the special cases of non-degenerate configurations (i.e., the cases where e.g. no three points lie on a line) and low dimensions (i.e., $d=2$ or $d=3$ ). We generate the entire list of all abstract order types and dissection types (i.e., we also list non-realizable cases) for small $n$, including also degenerate cases in arbitrary dimension $d$. The realizability problem in general is not addressed in this article; however, if $n$ is small enough then all instances are realizable (cf. Section 3.4). Although the realizability problem is very hard and needs further investigation, the complete generation of the abstract combinatorial types of point configurations and hyperplane arrangements offers a powerful database for various investigations (e.g., see Section 4).

This article is the full paper version of the extended abstract presented in [FF01]. A database of computational results can be accessed via the Internet on http://www.om.math.ethz.ch.

\section{Combinatorial Types and Oriented Matroids}

This section discusses order types and dissection types and their relation to oriented matroids.

\subsection{Order Types of Point Configurations}

Consider a point configuration $\mathcal{P}=\left\{v^{1}, \ldots, v^{n}\right\}$ of $n$ points in $\mathbb{R}^{d}$. An oriented hyperplane $H$ partitions $\mathcal{P}$ into three (possibly empty) sets $\mathcal{P}^{+}$, $\mathcal{P}^{-}, \mathcal{P}^{0}$ and defines a corresponding sign vector in $\{-,+, 0\}^{n}$, see Figure 1. The collection of all possible sign vectors obtained by hyperplanes from $\mathcal{P}$

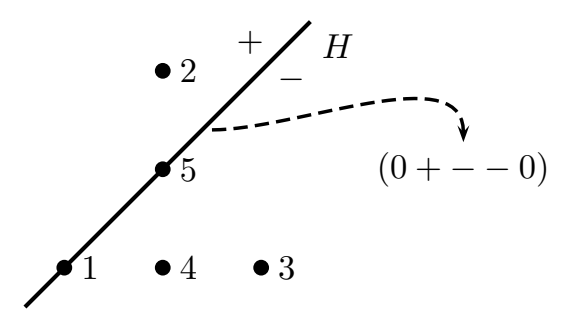

Figure 1: Sign vector defined by a hyperplane in a point configuration

defines the order type of $\mathcal{P}$. More formally, for $x \in \mathbb{R}^{d+1}$ define a sign vector $X$ by $X_{e}=\operatorname{sign}\left(\sum_{i=1}^{d+1} v_{i}^{e} x_{i}\right)$ for $e \in E:=\{1, \ldots, n\}$, where $v_{d+1}^{e}:=1$. Let $A$ be the matrix of the $n=|E|$ row vectors $v^{e} \in \mathbb{R}^{d+1}, e \in E$, and set $\mathcal{F}(\mathcal{P}):=\left\{\operatorname{sign}(A x) \mid x \in \mathbb{R}^{d+1}\right\}$. Two point configurations $\mathcal{P}$ and $\mathcal{P}^{\prime}$ are 
combinatorially equivalent if the sets of sign vectors $\mathcal{F}(\mathcal{P})$ and $\mathcal{F}\left(\mathcal{P}^{\prime}\right)$ are the same after possibly relabeling the points.

Definition 1 (Order Type of a Point Configuration). Consider a point configuration $\mathcal{P}=\left\{v^{e} \mid e \in E\right\}$ and $\mathcal{F}(\mathcal{P})$ as described above. The order type of $\mathcal{P}$ is defined as the relabeling class $\operatorname{LC}(E, \mathcal{F}(\mathcal{P}))$ of $(E, \mathcal{F}(\mathcal{P}))$, which is the set of all $\left(E^{\prime}, \mathcal{F}^{\prime}\right)$ that are obtained from $(E, \mathcal{F}(\mathcal{P}))$ by relabeling the elements in $E$.

The set $\mathcal{F}(\mathcal{P})$ is known as (the set of covectors of) a realizable oriented matroid: A pair $(E, \mathcal{F})$ is called a realizable oriented matroid if $E$ is a finite set and $\mathcal{F}=\{\operatorname{sign}(y) \mid y \in V\}$ for some vector subspace $V$ of $\mathbb{R}^{E}$. In general, oriented matroids are defined by a set of properties which hold for any $\mathcal{F}(\mathcal{P})$ (see Section 3$)$. Note that for any point configuration the sign vector $(+\cdots+)$ is in $\mathcal{F}(\mathcal{P})$. An oriented matroid $(E, \mathcal{F})$ such that $(+\cdots+) \in \mathcal{F}$ is called an acyclic oriented matroid.

The above definition of an order type is what we initially have described. The notion of order types has been introduced by Goodman and Pollack [GP83] using $\lambda$-functions, first for labeled point configurations and in the further discussion also for unlabeled point configurations. It was pointed out in [GP83] that the definitions of order types by $\lambda$-functions and oriented matroids are equivalent.

The one-to-one correspondence between all order types and all relabeling classes of realizable acyclic oriented matroids can be illustrated by use of oriented sphere arrangements which represent realizable oriented matroids. As before, we embed $\mathcal{P}$ in $\mathbb{R}^{d+1}$ by adding $v_{d+1}^{e}=1$ to every $v^{e}$. The extended vectors from $\mathcal{P}$ are the normal vectors of a central arrangement of hyperplanes. Its intersection with the unit sphere $S^{d}$ defines a sphere arrangement $\mathcal{S}$ as depicted in Figure 2, where every sphere in $\mathcal{S}$ is oriented

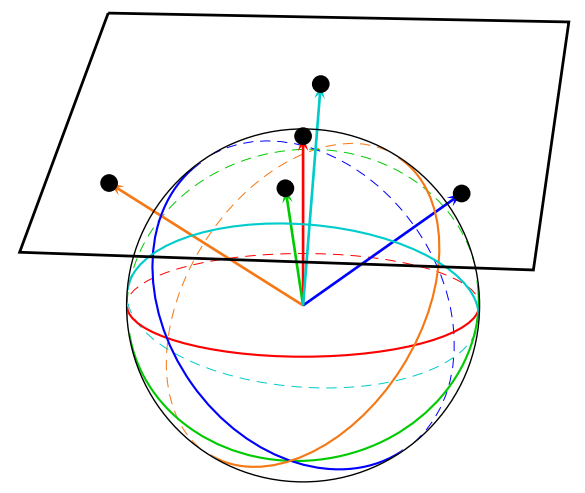

Figure 2: Point configuration and sphere arrangement 
according to its normal vector. Every cell in the sphere arrangement $\mathcal{S}$ has a one-to-one relation to a sign vector in $\mathcal{F}(\mathcal{P})$ as introduced above. The cell containing $v=(0, \ldots, 0,1)$ corresponds to the sign vector $(+\cdots+) \in \mathcal{F}(\mathcal{P})$.

A point configuration is non-degenerate, i.e., the points are in general position, if and only if the corresponding acyclic oriented matroid is uniform (in a corresponding sphere arrangement in $\mathbb{R}^{d}$ this means that no $d$ spheres intersect in a common point).

\subsection{Dissection Types of Hyperplane Arrangements}

Let $\mathcal{Q}=\left\{h^{1}, \ldots, h^{n}\right\}$ be a hyperplane arrangement in $\mathbb{R}^{d}$. For $e \in\{1, \ldots, n\}$ let $h^{e}$ be described by $v^{e} \in \mathbb{R}^{d+1}$ such that $h^{e}$ is the set of points $x \in \mathbb{R}^{d}$ for which $\sum_{i=1}^{d+1} v_{i}^{e} x_{i}=0$, where $x_{d+1}:=1$. Let $A$ be the matrix of the $n+1$ row vectors $v^{e} \in \mathbb{R}^{d+1}$ for $e \in E:=\{1, \ldots, n\} \cup\{g\}$, where $v^{g} \in \mathbb{R}^{d+1}$ is defined by $v_{d+1}^{g}:=1$ and $v_{i}^{g}:=0$ otherwise; $g \in E$ is a new index element which is called the infinity element. We define $\mathcal{F}(\mathcal{Q}):=\left\{\operatorname{sign}(A x) \mid x \in \mathbb{R}^{d+1}\right\}$ which is the set of covectors of a realizable oriented matroid (cf. Section 2.1).

The orientation of the hyperplanes $H_{e}$ has been arbitrarily determined by the choice of $v^{e}$. Therefore, two hyperplane arrangements $\mathcal{Q}$ and $\mathcal{Q}^{\prime}$ are combinatorially equivalent if $\mathcal{F}(\mathcal{Q})$ and $\mathcal{F}\left(\mathcal{Q}^{\prime}\right)$ are the same after possibly reorienting and relabeling the elements such that the infinity elements are identified.

Definition 2 (Dissection Type of a Hyperplane Arrangement). Let $\mathcal{Q}$ be a hyperplane arrangement, $g \in E$ the infinity element, and $\mathcal{F}(\mathcal{Q})$ as above. The dissection type of $\mathcal{Q}$ is the affine isomorphism class $\operatorname{AC}(E, \mathcal{F}(\mathcal{Q}), g)$, which is the set of all $\left(E^{\prime}, \mathcal{F}^{\prime}, g^{\prime}\right)$ that are obtained from $(E, \mathcal{F}(\mathcal{Q}), g)$ by isomorphisms which map $g$ to $g^{\prime}$, where an isomorphism is the composition of a reorientation (i.e., for some $S \subseteq E$ each $X \in \mathcal{F}$ is replaced by $X^{\prime}$ for which $X_{e}^{\prime}=-X_{e}$ if $e \in S$ and $X_{e}^{\prime}=X_{e}$ otherwise) and relabeling of elements.

We introduce the notion of dissection types of hyperplane arrangements in analogy to the notion of order types of point configurations.

For an oriented matroid $(E, \mathcal{F})$ and $g \in E$ we call the triple $(E, \mathcal{F}, g)$ an affine oriented matroid. The correspondence between dissection types and realizable affine oriented matroids can be illustrated similarly as for point configurations (see Section 2.1). The hyperplane arrangement $\mathcal{Q}$, embedded in $\mathbb{R}^{d+1}$ by adding a coordinate $x_{d+1}=1$, defines a sphere arrangement $\mathcal{S}$ on the unit sphere, where an extra sphere with normal vector $(0, \ldots, 0,1)$ is specially marked and corresponds to the infinity element (see Figure 3).

A hyperplane arrangement is non-degenerate, i.e., the hyperplanes are in general position (which also means that there are no parallel hyperplanes), if and only if the corresponding affine oriented matroid is uniform. 


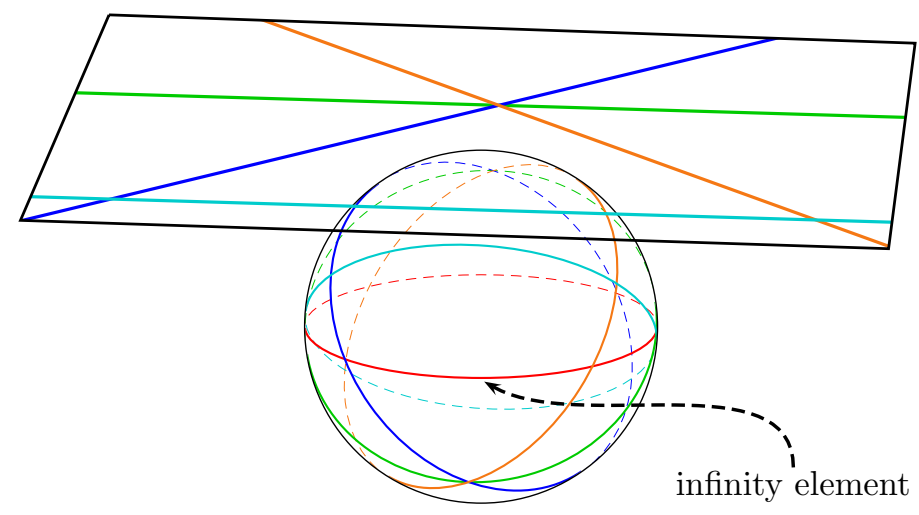

Figure 3: Hyperplane arrangement and sphere arrangement

\subsection{Oriented Matroids and Abstract Combinatorial Types}

Realizable oriented matroids correspond to sets of sign vectors defined by real vector subspaces or sphere arrangements. The following set (F0) to (F3) of properties are satisfied by realizable oriented matroids as introduced above; they are used as the axioms which define oriented matroids. Then oriented matroids are representable by topological sphere arrangements (also called pseudosphere arrangements) [FL78, Man82].

Definition 3 (Oriented Matroid). An oriented matroid $\mathcal{M}$ is a pair $(E, \mathcal{F})$ of a finite set $E$ and a set $\mathcal{F} \subseteq\{-,+, 0\}^{E}$ of sign vectors (called covectors) for which the following covector axioms (F0) to (F3) are valid:

$(\mathrm{F} 0)(0 \cdots 0) \in \mathcal{F}$.

(F1) If $X \in \mathcal{F}$ then $-X \in \mathcal{F}$.

(F2) If $X, Y \in \mathcal{F}$ then $X \circ Y \in \mathcal{F}$, where $(X \circ Y)_{e}:=X_{e}$ if $X_{e} \neq 0$ and $(X \circ Y)_{e}:=Y_{e}$ otherwise.

(F3) For all $X, Y \in \mathcal{F}$ and $e \in D(X, Y):=\left\{e \in E \mid X_{e}=-Y_{e} \neq 0\right\}$ there exists $Z \in \mathcal{F}$ such that $Z_{e}=0$ and $Z_{f}=(X \circ Y)_{f}$ for all $f \in E \backslash D(X, Y)$.

We have seen above that the order types of point configurations correspond to the relabeling classes of realizable acyclic oriented matroids, and the dissection types of hyperplane arrangements to the affine isomorphism classes of realizable affine oriented matroids. It is straightforward to generalize these notions (cf. Definitions 1 and 2) to oriented matroids as follows: 
Definition 4 (Abstract Order Type, Abstract Dissection Type). We call the relabeling class of an acyclic oriented matroid an abstract order type. For an oriented matroid $(E, \mathcal{F})$ and $g \in E$ we call the affine isomorphism class $\mathrm{AC}(E, \mathcal{F}, g)$ an abstract dissection type. If the oriented matroid is uniform then the corresponding abstract order type (or abstract dissection type) is called non-degenerate.

\section{Generation of Combinatorial Types}

This section discusses the generation of complete listings of (abstract) order types and dissection types. For this generation we use the relations to oriented matroids as discussed in Section 2. It is not evident how one can design any finite method to generate all order types and dissection types directly, i.e., without an axiomatic abstraction such as oriented matroids.

The listings of combinatorial types are organized by number of elements (e.g., points) $n$ and dimension $d$. In order to avoid unnecessary redundancies, a point configuration $\mathcal{P}$ in $\mathbb{R}^{d}$ is listed only if all points are distinct and $\mathcal{P}$ is not contained in a $(d-1)$-dimensional affine subspace. Similarly, a hyperplane arrangement is listed only if all hyperplanes are distinct and their normal vectors are not contained in a $(d-1)$-dimensional affine subspace. Equivalently, we will only consider oriented matroids $(E, \mathcal{F})$ which are socalled simple, i.e., there are no loops (elements $e \in E$ such that $X_{e}=0$ for all $X \in \mathcal{F}$ ) and no parallel elements (elements $e, f \in E$ such that $X_{e}=X_{f}$ for all $X \in \mathcal{F}$ or $X_{e}=-X_{f}$ for all $X \in \mathcal{F}$ ). The dimension of a point configuration or hyperplane arrangement is reflected by the dimension of the corresponding oriented matroid which is equal to the dimension spanned by a corresponding (topological) sphere arrangement.

\subsection{Generation of Oriented Matroids}

For given integers $n$ and $d$ consider the complete list $\operatorname{IC}(n, d)$ of all simple oriented matroids on $n$ elements of dimension $d$ up to isomorphism, i.e., up to reorientation and relabeling. We may think of $\operatorname{IC}(n, d)$ as a list containing all types of unlabeled and unoriented topological sphere arrangements with $n$ spheres on $S^{d}$. We described in [FF02] several methods how $\operatorname{IC}(n, d)$ can be generated. The following is a short sketch of the main steps using (topological) sphere arrangements for illustration

Every arrangement of $n$ (topological) spheres can be generated from some arrangement $\mathcal{S}$ of $n-1$ spheres by inserting a new sphere $S_{n}$; this is called a single element extension. The oriented sphere $S_{n}$ defines for every vertex (i.e., 0 -dimensional intersection of spheres) of $\mathcal{S}$ a corresponding sign $(-,+$, or 0 ); the collection of all signs defined by a single element extension is called 
a localization. Localizations (and by this single element extensions) can be characterized by a result of Las Vergnas [LV78] using coline cycles, which are the 1-dimensional intersections of spheres in $\mathcal{S}$ : a collection of signs on the vertices of $\mathcal{S}$ is a localization if and only if the sign pattern of every coline cycle has one of the three types that are indicated in Figure 4. While the only-if
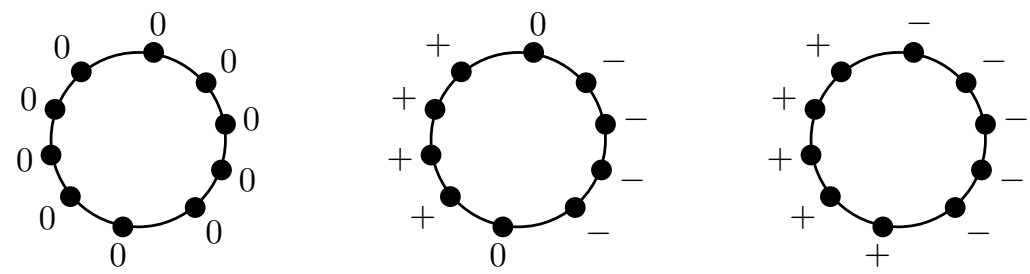

Figure 4: Sign patterns of coline cycles characterizing single element extension

part is obvious, the if part is nontrivial. This characterization can be used in a backtracking method which enumerates all single element extensions; there are efficient implementations of the algorithm using a dynamic ordering of the coline cycles in the backtracking process and a special data structure which encodes the mutual intersections of coline cycles.

By use of special canonical representations of isomorphism classes of oriented matroids (based on chirotopes representing basis orientations) and applying a reverse search technique [AF96], the generation methods can be designed so that they list the isomorphism classes without repetition in a canonical ordering (see [Fin01]). The expensive operation is the computation of canonical representations; our implementations have a worst case complexity of $O(n !)$. We hope that this can be improved significantly in the future. However, our methods reduce redundancies already while backtracking (using a coarse identity induced by symmetries of the given arrangement $\mathcal{S}$ ), so only relatively few canonical representations have to be computed.

Table 1 on the left shows the numbers $|\mathrm{IC}(n, d)|$ of oriented matroids up to isomorphism, and for comparison we give on the right hand side of Table 1 the corresponding numbers for the non-degenerate (i.e., uniform) cases only (these have been generated and discussed in former work; see Table 6 in [Bok93]). For $d \geq n$ there are blanks in the tables as there are too few elements to span the dimension. We will use the lists $\operatorname{IC}(n, d)$ as the input for the following.

\subsection{Generation of Abstract Order Types}

Consider an oriented sphere arrangement $\mathcal{S}$ in $\operatorname{IC}(n, d)$. The corresponding oriented matroid is acyclic if some cell $c$ of maximal dimension corresponds 


\begin{tabular}{|c|c|c|c|c|c|c|c|c|c|c|c|c|c|c|}
\hline & \multicolumn{7}{|c|}{ all oriented matroids } & \multicolumn{7}{|c|}{ uniform only } \\
\hline$n=$ & 3 & 4 & 5 & 6 & 7 & 8 & 9 & 3 & 4 & 5 & 6 & 7 & 8 & 9 \\
\hline$d=2$ & 1 & 2 & 4 & 17 & 143 & 4890 & 461053 & 1 & 1 & 1 & 4 & 11 & 135 & 4382 \\
\hline$d=3$ & & 1 & 3 & 12 & 206 & 181472 & $?$ & & 1 & 1 & 1 & 11 & 2628 & $?$ \\
\hline$d=4$ & & & 1 & 4 & 25 & 6029 & $?$ & & & 1 & 1 & 1 & 135 & $?$ \\
\hline$d=5$ & & & & 1 & 5 & 50 & 508321 & & & & 1 & 1 & 1 & 4382 \\
\hline$d=6$ & & & & & 1 & 6 & 91 & & & & & 1 & 1 & 1 \\
\hline$d=7$ & & & & & & 1 & 7 & & & & & & 1 & 1 \\
\hline$d=8$ & & & & & & & 1 & & & & & & & 1 \\
\hline
\end{tabular}

Table 1: Number of oriented matroids up to isomorphism

to the sign vector $X(c)=(+\cdots+)$; geometrically, we can assume (after an appropriate rotation of $\mathcal{S}$ ) that $c$ contains the vector $(0, \ldots, 0,1)$ (cf. Section 2.1). For an arbitrary cell $c$ of maximal dimension $\mathcal{S}$ in $\operatorname{IC}(n, d)$ a reorientation of $\mathcal{S}$ according to $X(c)$ will let $c$ correspond to $(+\cdots+)$. Hence the list of all sign vectors corresponding to cells of maximal dimension in $\mathcal{S}$ (known as topes), which we can compute efficiently, is sufficient to find all abstract order types isomorphic to $\mathcal{S}$. See Pseudo-Code 1 for an outline of the algorithm. Note that every abstract order type belongs to a unique isomorphism class of oriented matroids, hence every abstract order type is generated exactly once.

Input: $n, d$.

Output: all abstract order types with $n$ points and dimension $d$.

for every representative $\mathcal{M} \in \mathrm{IC}(n, d)$ do

compute the set of topes $\mathcal{T}$ of $\mathcal{M}$;

for every tope $X \in \mathcal{T}$ do

$\mathcal{M}^{X}:=$ reorientation of $\mathcal{M}$ according to $X$;

$\mathcal{M}_{\text {rep }}^{X}:=$ canonical representative in the relabeling class $\mathrm{LC}\left(\mathcal{M}^{X}\right)$; endfor;

after removing multiple entries, output the set $\left\{\mathcal{M}_{\text {rep }}^{X} \mid X \in \mathcal{T}\right\}$;

endfor.

Pseudo-Code 1: Generation of abstract order types

The left hand side of Table 2 shows the numbers of abstract order types obtained by computations. Note that there are considerably fewer nondegenerate abstract order types, i.e., abstract order types corresponding to uniform oriented matroids (see right hand side of Table 2); the numbers for $d=2$ coincide with the known results [Knu92, AAK01]. 


\begin{tabular}{|c|c|c|c|c|c|c|c|c|c|c|c|c|}
\hline & \multicolumn{6}{|c|}{ all abstract order types } & \multicolumn{6}{|c|}{ non-degenerate only } \\
\hline$n=$ & 34 & 5 & 6 & 7 & 8 & 9 & 34 & 5 & 6 & 7 & 8 & $\overline{9}$ \\
\hline$\overline{d=2}$ & 13 & 11 & 93 & 2121 & 122508 & 15296266 & 12 & 31 & 16 & 35 & 3315 & 158830 \\
\hline$d=3$ & 1 & 5 & 55 & 5083 & 10775236 & ? & 1 & 2 & 4 & 246 & 160020 & ? \\
\hline$d=4$ & & 1 & 8 & 204 & 505336 & ? & & 1 & 3 & 8 & 11174 & ? \\
\hline$d=5$ & & & 1 & 11 & 705 & ? & & & 1 & 3 & 11 & 938513 \\
\hline$d=6$ & & & & 1 & 15 & 2293 & & & & 1 & 4 & 22 \\
\hline$d=7$ & & & & & 1 & 19 & & & & & 1 & 4 \\
\hline$d=8$ & & & & & & 1 & & & & & & 1 \\
\hline
\end{tabular}

Table 2: Number of abstract order types

\subsection{Generation of Abstract Dissection Types}

If in a realizable oriented matroid $\mathcal{M}$ an element $g$ is marked as the infinity element, then there exists a representation by a sphere arrangement $\mathcal{S}$ where the sphere corresponding to $g$ has the normal vector $(0, \ldots, 0,1)$. Analogously, the complete list of abstract dissection types for $n$ hyperplanes in $\mathbb{R}^{d}$ is obtained from $\operatorname{IC}(n+1, d)$ by marking infinity elements in all possible ways and by identifying affine isomorphic instances. Pseudo-Code 2 summarizes the algorithm. Every abstract dissection type belongs to a unique isomorphism class of oriented matroids, hence every abstract dissection type is generated exactly once.

Input: $n, d$.

Output: all abstract dissection types with $n$ hyperplanes and dimension $d$.

for every representative $\mathcal{M}=(E, \mathcal{F}) \in \operatorname{IC}(n+1, d)$ do

for every element $g \in E=\{1, \ldots, n+1\}$ do

$\mathcal{M}_{\text {rep }}^{g}:=$ canonical representative in the affine isomorphism class $\operatorname{AC}(E, \mathcal{F}, g)$;

endfor;

after removing multiple entries, output the set $\left\{\mathcal{M}_{\text {rep }}^{g} \mid g \in E\right\}$;

endfor.

Pseudo-Code 2: Generation of abstract dissection types

The numbers of abstract dissection types obtained by computations can be found in Table 3 on the left. For comparison, the right hand side of Table 3 shows corresponding numbers for non-degenerate dissection types; the known numbers (see [Rin56]) for $d=2$ and $n \leq 7$ coincide with the numbers obtained by our programs. 


\begin{tabular}{|c|c|c|c|c|c|c|c|c|c|c|c|c|c|c|}
\hline & \multicolumn{7}{|c|}{ all abstract dissection types } & \multicolumn{7}{|c|}{ non-degenerate only } \\
\hline$n+1=$ & 3 & 4 & 5 & 6 & 7 & 8 & 9 & 3 & 4 & 5 & 6 & 7 & 8 & $\overline{9}$ \\
\hline$d=2$ & 1 & 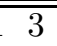 & 8 & 46 & 790 & 37829 & 4134939 & 1 & 1 & 1 & 6 & 43 & 922 & 38612 \\
\hline$d=3$ & & 1 & & 27 & 1063 & 1434219 & $?$ & & 1 & 1 & 1 & 43 & 20008 & $?$ \\
\hline$d=4$ & & & 1 & 7 & 71 & 44956 & $?$ & & & 1 & 1 & 1 & 922 & $?$ \\
\hline$d=5$ & & & & 1 & 9 & 156 & $?$ & & & & 1 & 1 & 1 & 38612 \\
\hline$d=6$ & & & & & 1 & 11 & 325 & & & & & 1 & 1 & 1 \\
\hline$d=7$ & & & & & & 1 & 13 & & & & & & 1 & 1 \\
\hline$d=8$ & & & & & & & 1 & & & & & & & 1 \\
\hline
\end{tabular}

Table 3: Number of abstract dissection types

\subsection{Notes on the Realizability}

The generation of combinatorial types often was considered together with the realizability problem, the problem of classifying which abstract types can be realized by coordinates in Euclidean space and which are not realizable.

For $d=2$ the classification of the uniform cases is due to Grünbaum [Grü67, Grü72] for $n=7$, Goodman and Pollack [GP80a] for $n=8$, RichterGebert [Ric88] and Gonzalez-Sprinberg and Laffaille [GSL89] for $n=9$, finally Bokowski, Laffaille, and Richter-Gebert (unpublished) for $n=10$; for $d=3$ and $n=8$ the classification is due to Bokowski and Richter-Gebert [BRG90]. The realizability problem is attacked from two sides: (i) finding realizations (using randomly generated points, various insertion or perturbation techniques) and (ii) proving that no realization can exist (e.g., with final polynomials [RG92]).

In the general case (which includes degenerate configurations) important questions are still open. It is not clear how the methods to detect nonrealizability of uniform instances can be generalized to the degenerate case. Furthermore, finding coordinates for realizable instances has the additional difficulty that no rational solution may exist. The classification problem for the general case is solved for $d=2$ and $n \leq 8$ due to Goodman and Pollack [GP80b] (all cases are realizable, which was a conjecture of Grünbaum [Grü72]). By duality, all cases are realizable for $n \leq 8$ in any dimension except $d=3$, where the first non-realizable cases occur already for $n=8$. Also by duality, all instances with $n-d \leq 3$ are realizable. Hence, our computational results contain non-realizable cases only in the lists for $(n, d)$ equal to $(8,3)$, $(9,2)$, and $(9,5)$; here, the number of non-realizable uniform isomorphism classes of oriented matroids are 24, 1, and 1, respectively. Furthermore, it is known that for $(n, d)=(10,2)$ there are 312356 isomorphism classes whereof 242 are non-realizable. W.r.t. non-degenerate abstract order types it is known that for $(n, d)=(9,2)$ there are 13 non-realizable types, and for $(n, d)=(10,2)$ there are 10635 non-realizable types out of 14320182 abstract 
types [AAK01]. Corresponding numbers for degenerate non-realizable types are not known.

We present in Figures 5 to 7 realizations of abstract order types for small instances in $\mathbb{R}^{2}$, i.e., for configurations of 3 to 6 points. The trivial types of collinear points (i.e., all points on a line) correspond to combinatorial types in $\mathbb{R}^{1}$ and are not counted in $\mathbb{R}^{2}$. We draw the point configurations in the figures with some lines which may be helpful when reading the picture. The lines which we added are marking a non-degeneracy (i.e., three or more collinear points) and the convex hull, and if there are points in the interior of the convex hull, all lines through the points on the boundary of the convex hull (extreme points) are shown; the rule is applied recursively after removing the extreme points.
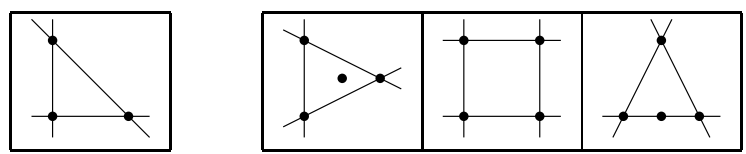

Figure 5: The order types with 3 and 4 non-collinear points in $\mathbb{R}^{2}$

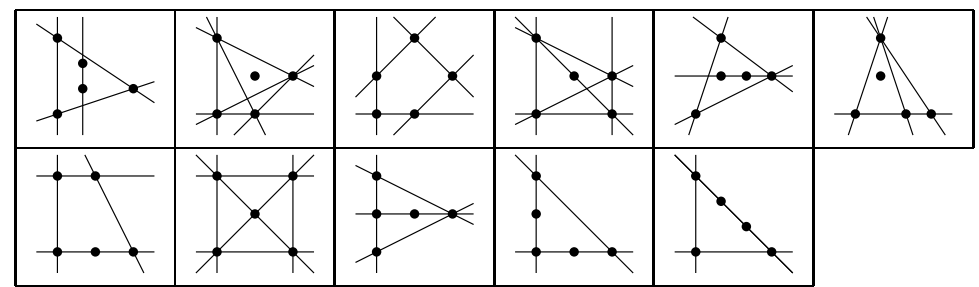

Figure 6: The 11 order types with 5 non-collinear points in $\mathbb{R}^{2}$; only the first 3 are non-degenerate

We present in Figures 8 to 10 realizations of abstract dissection types for small instances in $\mathbb{R}^{2}$, i.e., for arrangements of 2 to 5 hyperplanes. Degenerate intersections (i.e., points where three or more lines intersect) are marked; lines without intersection in the drawing are parallel. The trivial types of all lines parallel correspond to combinatorial types in $\mathbb{R}^{1}$ and are not counted in $\mathbb{R}^{2}$.

\section{Applications}

Before we discuss one example in more detail below, the following few remarks may hint on some possible impacts of our database of combinatorial types. There has been a strong interest in the number of faces ( $f$-vectors) and specially the number of simplicial topes (mutations) of arrangements and ori- 


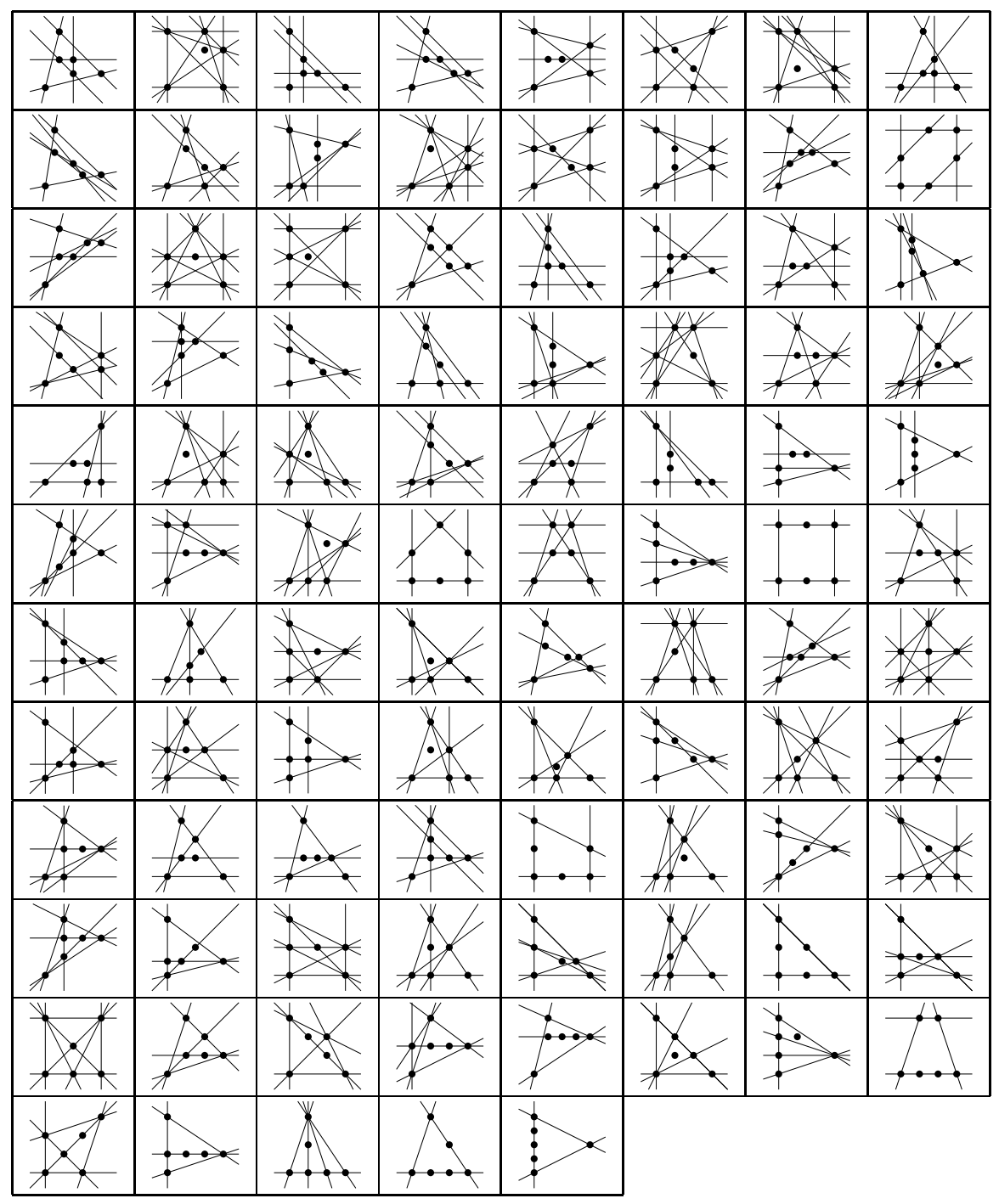

Figure 7: The 93 order types with 6 non-collinear points in $\mathbb{R}^{2}$; only the first 16 are non-degenerate
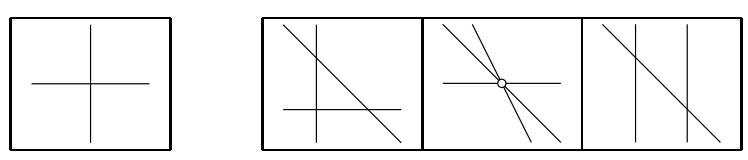

Figure 8: The dissection types with 2 and 3 non-parallel hyperplanes in $\mathbb{R}^{2}$ 


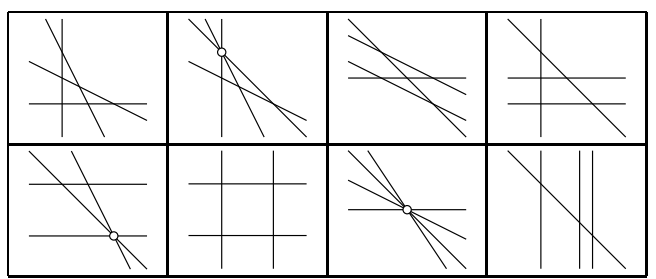

Figure 9: The 8 dissection types with 4 non-parallel hyperplanes in $\mathbb{R}^{2}$; only the first is non-degenerate

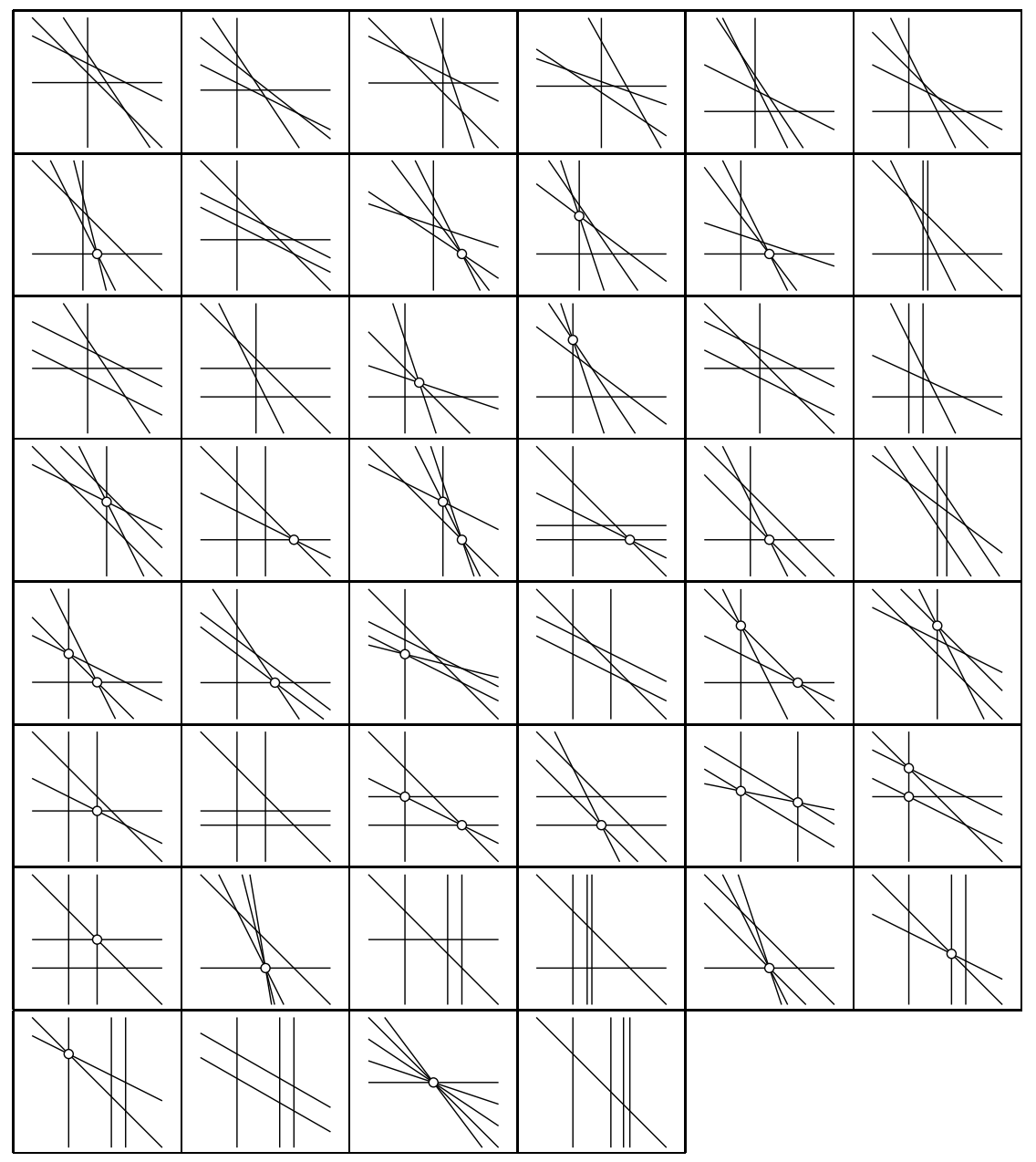

Figure 10: The 46 dissection types with 5 non-parallel hyperplanes in $\mathbb{R}^{2}$; only the first 6 are non-degenerate 
ented matroids, or in $k$-sets and extremal properties of point configurations; our database provides all these data for further investigations. For previous results based on listings of (non-degenerate) order types see also [AK01].

The list of abstract order types has been used to compute all (abstract) types of convex polytopes which coincide with the known numbers of combinatorial types of polytopes (e.g., see [KK95]), by this providing an independent proof of these results.

Consider the following conjecture of da Silva and Fukuda (Conjecture 4.2 in [dSF98]), which is a strong version of the Sylvester-Gallai Theorem: Let $\mathcal{P}$ be a point configuration in $\mathbb{R}^{2}$, not all points on a line. Let $H$ be a line which does not contain a point from $\mathcal{P}$ but separates $\mathcal{P}$ into two parts $\mathcal{P}^{-}$ and $\mathcal{P}^{+}$such that $\left|\mathcal{P}^{-}\right|$and $\left|\mathcal{P}^{+}\right|$differ by at most 1 . Then there exists a line $\tilde{H}$ which contains exactly two points of $\mathcal{P}$, one from $\mathcal{P}^{-}$and one from $\mathcal{P}^{+}$.

Some weaker versions of this conjecture have been proved by Pach and Pinchasi [PP00]. We have tested the conjecture itself against our database of abstract order types: It is valid for $n \leq 8$ points, but for $n=9$ points the list of 15296266 abstract order types contains one counter-example, and this is the only one for $n=9$. Moreover this abstract order type has been found to be realizable; a picture of the counter-example is given in Figure 11.

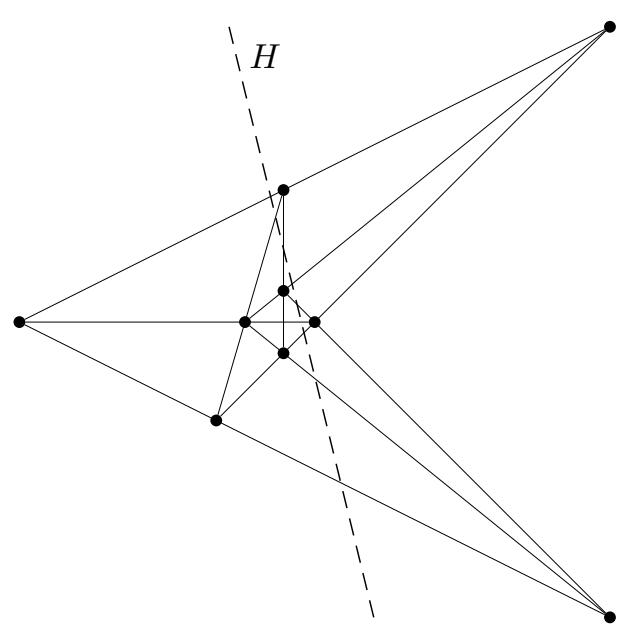

\begin{tabular}{|c|c|}
\hline \multicolumn{2}{|c|}{ Coordinates } \\
$x$ & $y$ \\
\hline \hline 1 & 1 \\
\hline$\frac{1}{2}$ & 0 \\
\hline$\frac{1}{\sqrt{5}}$ & $-1+\frac{2}{\sqrt{5}}$ \\
\hline$\frac{1}{3}$ & $-\frac{1}{3}$ \\
\hline$\frac{3}{2}-\frac{\sqrt{5}}{2}$ & 0 \\
\hline$\frac{1}{\sqrt{5}}$ & $1-\frac{2}{\sqrt{5}}$ \\
\hline 0 & 0 \\
\hline$\frac{1}{\sqrt{5}}$ & $\frac{1}{\sqrt{5}}$ \\
\hline 1 & -1 \\
\hline
\end{tabular}

Figure 11: The counter-example to the conjecture of da Silva and Fukuda with 9 points 


\section{References}

[AAK01] O. Aichholzer, F. Aurenhammer, and H. Krasser. Enumerating order types for small point sets with applications. In Proc. 17th Ann. ACM Symp. Comput. Geometry, pages 11-18. Medford, MA, USA, 2001.

[AF96] D. Avis and K. Fukuda. Reverse search for enumeration. Discrete Appl. Math., 65(1-3):21-46, 1996.

[AK01] O. Aichholzer and H. Krasser. The point set order type data base: a collection of applications and results. In Thirteenth Canadian Conference on Computational Geometry, pages 17-20. University of Waterloo, 2001.

[BGdO00] J. Bokowski and A. Guedes de Oliveira. On the generation of oriented matroids. Discrete Comput. Geom., 24(2-3):197-208, 2000.

[BLVS $\left.{ }^{+} 99\right]$ A. Björner, M. Las Vergnas, B. Sturmfels, N. White, and G. M. Ziegler. Oriented matroids. Cambridge University Press, Cambridge, second edition, 1999.

[Bok93] J. Bokowski. Oriented matroids. In Handbook of convex geometry, Vol. A, B, pages 555-602. North-Holland, Amsterdam, 1993.

[BRG90] J. Bokowski and J. Richter-Gebert. On the classification of non-realizable oriented matroids. Preprint Nr. 1345, Technische Hochschule, Darmstadt.

[dSF98] I. P. F. da Silva and K. Fukuda. Isolating points by lines in the plane. J. Geom., 62(1-2):48-65, 1998.

[FF01] L. Finschi and K. Fukuda. Complete combinatorial generation of small point configurations and hyperplane arrangements. In Thirteenth Canadian Conference on Computational Geometry, pages 97-100. University of Waterloo, 2001.

[FF02] L. Finschi and K. Fukuda. Generation of oriented matroids a graph theoretical approach. Discrete Comput. Geom., 27:117$136,2002$.

[Fin01] L. Finschi. A Graph Theoretical Approach for Reconstruction and Generation of Oriented Matroids. Ph.D. thesis, Swiss Federal Institute of Technology ETH Zürich, 2001.

[FL78] J. Folkman and J. Lawrence. Oriented matroids. J. Combin. Theory Ser. B, 25(2):199-236, 1978. 
[GP80a] J. E. Goodman and R. Pollack. On the combinatorial classification of nondegenerate configurations in the plane. J. Combin. Theory Ser. A, 29(2):220-235, 1980.

[GP80b] J. E. Goodman and R. Pollack. Proof of Grünbaum's conjecture on the stretchability of certain arrangements of pseudolines. $J$. Combin. Theory Ser. A, 29(3):385-390, 1980.

[GP83] J. E. Goodman and R. Pollack. Multidimensional sorting. SIAM J. Comput., 12(3):484-507, 1983.

[GP84] J. E. Goodman and R. Pollack. Semispaces of configurations, cell complexes of arrangements. J. Combin. Theory Ser. A, 37(3):257-293, 1984.

[Grü67] B. Grünbaum. Convex polytopes. Interscience Publishers John Wiley \& Sons, Inc., New York, 1967.

[Grü72] B. Grünbaum. Arrangements and spreads. American Mathematical Society Providence, R.I., 1972.

[GSL89] G. Gonzalez-Sprinberg and G. Laffaille. Sur les arrangements simples de huit droites dans $\mathbf{R P}^{2}$. C. R. Acad. Sci. Paris Sér. I Math., 309(6):341-344, 1989.

[KK95] V. Klee and P. Kleinschmidt. Convex polytopes and related complexes. In Handbook of combinatorics, Vol. 1, 2, pages 875-917. Elsevier, Amsterdam, 1995.

[Knu92] D. E. Knuth. Axioms and hulls, volume 606 of Lecture Notes in Computer Science. Springer-Verlag, Berlin, 1992.

[LV78] M. Las Vergnas. Extensions ponctuelles d'une géométrie combinatoire orientée. In Problèmes combinatoires et théorie des graphes (Colloq. Internat. CNRS, Univ. Orsay, Orsay, 1976), pages 265-270. CNRS, Paris, 1978.

[Man82] A. Mandel. Topology of oriented matroid. Ph.D. thesis, University of Waterloo, 1982.

[Mnë88] N. E. Mnëv. The universality theorems on the classification problem of configuration varieties and convex polytopes varieties. In Topology and geometry - Rohlin Seminar, volume 1346 of Lecture Notes in Math., pages 527-543. Springer, Berlin, 1988.

[PP00] J. Pach and R. Pinchasi. Bichromatic lines with few points. $J$. Combin. Theory Ser. A, 90(2):326-335, 2000.

[RG92] J. Richter-Gebert. On the realizability problem of combinatorial geometries decision methods. Ph.D. thesis, TU Darmstadt, 1992. 
[Ric88] J. Richter. Kombinatorische Realisierbarkeitskriterien für orientierte Matroide. Master's thesis, Technische Hochschule, Darmstadt, 1988. published in Mitteilungen Mathem. Seminar Giessen, Heft 194, Giessen 1989.

[Rin56] G. Ringel. Teilungen der Ebene durch Geraden oder topologische Geraden. Math. Z., 64:79-102, 1956.

[Sho91] P. W. Shor. Stretchability of pseudolines is NP-hard. In P. Gritzmann and B. Sturmfels, editors, Applied geometry and discrete mathematics: the Victor Klee Festschrift, volume 4 of DIMACS series in discrete mathematics and theoretical computer science, pages 531-554. Amer. Math. Soc., Providence, RI, 1991.

\section{About Authors}

Lukas Finschi is at the Institute for Operations Research, Swiss Federal Institute of Technology, Zurich, Switzerland; finschi@ifor.math.ethz.ch. Komei Fukuda is at the Institute for Operations Research, Swiss Federal Institute of Technology, Zurich, Switzerland and Department of Mathematics, Swiss Federal Institute of Technology, Lausanne; fukuda@ifor.math.ethz.ch.

\section{Acknowledgments}

Work on this paper has been partially supported by the Swiss National Science Foundation Grant 21-58977.99. 